\title{
SUBALGEBRAS, DIRECT PRODUCTS AND ASSOCIATED LATTICES OF MV-ALGEBRAS
}

\author{
by L. P. BELLUCE, A. DI NOLA and A. LETTIERI
}

(Received 3 April, 1991)

0. Introduction. MV-algebras were introduced by C. C. Chang [3] in 1958 in order to provide an algebraic proof for the completeness theorem of the Lukasiewicz infinite valued propositional logic. In recent years the scope of applications of MV-algebras has been extended to lattice-ordered abelian groups, AF $\mathrm{C}^{*}$-algebras $[\mathbf{1 0}]$ and fuzzy set theory [1].

In [1] Belluce defined a functor $\gamma$ from MV-algebras to bounded distributive lattices; this functor was used in proving a representation theorem and was also used to show that the prime ideal space of an MV-algebra is homeomorphic to the prime ideal space of some bounded distributive lattice (both spaces endowed with the Stone topology). The problem of what the range of $\gamma$ is arises naturally. This question bears a relation to the question as to whether there is an "MV-space" in the same manner as there are Boolean spaces for Boolean algebras. Some "MV-spaces" are considered by N. G. Martinez [9].

A study of this problem was begun by Cignoli, Di Nola and Lettieri [6] where it was shown that certain elements in the range of $\gamma$ have a direct decomposition by linear elements in the same range. In [2] it is proved that some bounded countable chains are in the range of $\gamma$; moreover a least $\mathrm{MV}$-algebra $A$ for which $\gamma(A)$ is a given bounded countable chain is presented.

In this paper we examine the action of $\gamma$ on direct products and subalgebras of MV-algebras. We operate in an extended category of pairs $(A, \mathscr{I})$ where $A$ is an $\mathrm{MV}$-algebra and $\mathscr{I}$ a non-empty set of prime ideals. We show that this category has product and that $\gamma$ commutes with products. Under certain conditions we show that $\gamma$ preserves monomorphisms. We also give a necessary condition for a bounded distributive lattice to be in the range of $\gamma$, from which it follows that not every such lattice is in the range of $\gamma$. And finally, we show that $[0,1]$, as a lattice, is in the range of $\gamma$, as well as every complete bounded chain.

For the basic definition and properties of $\mathrm{MV}$-algebras the reader is referred to $[1],[2],[3],[10]$.

We consider an extended category $\mathscr{E}_{\mathrm{MV}}$ of MV-algebras. The objects of $\mathscr{E}_{\mathrm{MV}}$ are pairs $(A, \mathscr{I})$ where $A$ is an $\mathrm{MV}$-algebra and $\mathscr{I}$ a non-empty subset of $\operatorname{Spec} A$, the set of prime ideals of $A$; a morphism $f:\left(A_{1}, \mathscr{I}_{1}\right) \rightarrow\left(A_{2}, \mathscr{I}_{2}\right)$ of $\mathscr{E}_{\mathrm{MV}}$ is an MV-homomorphism $f: A_{1} \rightarrow A_{2}$ such that $f^{-1}\left(\mathscr{I}_{2}\right) \subseteq \mathscr{I}_{1}$, i.e. if $Q \in \mathscr{I}_{2}$ then $f^{-1}(Q) \in \mathscr{I}_{1}$.

From [1] we have a functor $\gamma: \mathscr{E}_{\mathrm{MV}} \rightarrow \mathscr{D}$ where $\mathscr{D}$ is the category of distributive lattices with 0,1 . The lattice $\gamma(A, \mathscr{I})$ has as elements equivalence classes $[x]_{\mathscr{I}}, x \in A$, where $[x]_{\mathscr{I}}=[y]_{\mathscr{S}}$ if for all $P \in \mathscr{I}, x \in P$ iff $y \in P$. Then $[x]_{\mathscr{I}}+[y]_{\mathscr{P}}=[x+y]_{\mathscr{S}},[x]_{\mathscr{S}}[y]_{\mathscr{S}}=$ $[x \wedge y]_{\mathscr{F}}$ are well-defined operations and $\gamma(A, \mathscr{I})$ becomes a distributive lattice with $0=[0]_{\mathscr{g}}$ and $1=[1]_{\mathscr{J}}$. If $f:\left(A_{1}, \mathscr{I}_{1}\right) \rightarrow\left(A_{2}, \mathscr{I}_{2}\right)$ is an $\mathscr{E}_{\mathrm{MV}}$-morphism then $\gamma(f): \gamma\left(A_{1}, \mathscr{J}_{1}\right) \rightarrow$ $\gamma\left(A_{2}, \mathscr{I}_{2}\right)$ is the lattice homomorphism, $\gamma(f)[x]_{\mathscr{F}_{1}}=[f(x)]_{\mathscr{I}_{2}} \cdot \gamma(f)$ is an epimorphism if $f$ is. $\gamma(A, \mathscr{I})$ is denoted by $[A]_{\mathscr{I}}$, or, when $\mathscr{I}=\operatorname{Spec} A$, by $[A]$.

The main features about $\gamma(A, \mathscr{I})$ are that some of its structure is reflected in $A$ and its ideal structure parallels that of $A$; in particular $\operatorname{Spec} A, \operatorname{Spec}[A]$ are homeomorphic.

Glasgow Math. J. 34 (1992) 301-307. 
1. In this first section we show the range of $\gamma$ is a proper subclass of $\mathscr{D}$.

Let $\mathscr{A}$ be an MV-algebra or a distributive lattice with 0,1 . We shall say that $\mathscr{A}$ has the prime-extension property (pep) if whenever $I \subseteq J$ are proper ideals of $\mathscr{A}$ and $I$ is prime then $J$ is prime. We shall show that $\gamma$ preserves pep.

First we recall that in [11, Chapter III, §6, Prop. 3] it is shown that the prime deductive systems containing a given prime deductive system form a chain; so we surely can say that:

Theorem 1.1. Every MV-algebra A has pep.

THEOREM 1.2. [A] has pep.

TheOREM 1.3. Let $\mathscr{L}_{1}, \mathscr{L}_{2} \in \mathscr{D} ;$ let $g: \mathscr{L}_{1} \rightarrow \mathscr{L}_{2}$ be an epimorphism. Then if $\mathscr{L}_{1}$ has pep so does $\mathscr{L}_{2}$.

Proof. Let $L \subseteq S$ be proper ideals of $\mathscr{L}_{2}$ with $L$ prime. Then $g^{-1}(L) \subseteq g^{-1}(S)$ and both are proper ideals of $\mathscr{L}_{1}$. But $g^{-1}(L)$ is prime, hence $g^{-1}(S)$ is prime. Let $a b \in S . g$ is an epimorphism so there are $x, y \in \mathscr{L}_{1}$ with $g(x)=a, g(y)=b$. Hence $g(x y) \in S$ so $x y \in g^{-1}(S)$. Thus $x \in g^{-1}(S)$ or $y \in g^{-1}(S)$ and it follows that $a \in S$ or $b \in S$, so $S$ is prime.

Now let $A$ be an MV-algebra, $\mathscr{I} \subseteq \operatorname{Spec} A, \mathscr{I} \neq \varnothing$. We clearly have an epimorphism $i:(A, \operatorname{Spec} A) \rightarrow(A, \mathscr{I})$ in $\mathscr{E}_{\mathrm{MV}}: i(x)=x$. Thus we have an epimorphism, $[A] \rightarrow[A]_{\mathscr{H}}$, $[x] \rightarrow[x]_{\Phi}$. By Theorems $1.2,1.3$ we have the following result.

Theorem 1.4. For every $(A, \mathscr{I}) \in \mathscr{E}_{\mathrm{MV}},[A]_{\mathscr{I}}$ has pep.

Thus a necessary condition for a bounded distributive lattice to lie in the range of $\gamma$ is for it to have pep. Since there exist distributive lattices with 0,1 that do not have pep, we have

THEOREM 1.5. The image of $\gamma: \mathscr{E}_{\mathrm{MV}} \rightarrow \mathscr{D}$ is a proper subclass of $\mathscr{D}$.

A bounded distributive lattice is called a $P_{m}$-lattice if each prime ideal is contained in a unique maximal ideal [8].

Theorem 1.6. Let $\mathscr{L}$ be a bounded distributive lattice with pep. Then $\mathscr{L}$ is a $P_{m}$-lattice.

Proof. Let $P$ a prime ideal of $\mathscr{L}, M_{1}, M_{2}$ maximal ideals and assume $P \subseteq M_{1}, P \subseteq M_{2}$. Suppose $M_{1} \neq M_{2}$ : Choose $a \in M_{1}-M_{2}, b \in M_{2}-M_{1}$. Then $a b \in M_{1} \cap M_{2}$ is prime. Thus $a \in M_{1} \cap M_{2}$ or $b \in M_{1} \cap M_{2}$, both impossible since $a \notin M_{2}$ and $b \notin M_{i}$. Thus $M_{1}=M_{2}$. space.

By Corollary 1.3 of [8] the maximal ideal space of a pep lattice $\mathscr{L}$ is a Hausdorff

Corollary 1.1. Any lattice in the range of $\gamma$ is a $P_{m}$-lattice, and so also has a Hausdorff maximal ideal space.

By [1, Theorems 15 and 20] we now have

Corollary 1.2. The maximal ideal space of an MV-algebra $A$ is Hausdorff.

2. Here we will show that $\mathscr{E}_{\mathrm{MV}}$ is closed order products and that $\gamma$ commutes with the taking of products. Thus we see that the image of $\gamma$ is closed under direct products. 
In this section $I$ will be an index set and for each $i \in I$ we have an object $\left(A_{i}, \mathscr{I}_{i}\right) \in \mathscr{E}_{\mathrm{MV}}$. Let $A=\prod_{i \in I} A_{i}$. For each $i \in I$ we have projections $\mathrm{pr}_{i}: A \rightarrow A_{i}$. If $i_{0} \in I$ and $Q \in \mathscr{I}_{i_{0}}$ then $\operatorname{pr}_{i_{0}}^{-1}(Q)=P$ is a prime ideal of $A$, call the ideal of $A$ over $Q$, which we will denote by $\operatorname{Ov}(Q)$. Let $\Phi=\left\{P\right.$ :for some $\left.Q \in \bigcup_{i \in I} \mathscr{I}_{i}, P=\operatorname{Ov}(Q)\right\}$. Then $\mathscr{I} \neq \varnothing$ and $\mathscr{I} \subseteq \operatorname{Spec} A$. Clearly then the maps $\operatorname{pr}_{i}:(A, \mathscr{I}) \rightarrow\left(A_{i}, \mathscr{I}_{i}\right)$ are $\mathscr{E}_{\mathrm{MV}}$ morphism. Now let $\left(A^{\prime}, \mathscr{I}^{\prime}\right)$ be an $\mathscr{C}_{\mathrm{MV}}$ morphism and such that for each $i \in I$ we have an $\mathscr{E}_{\mathrm{MV}}$ morphism $f_{i}:\left(A^{\prime}, \mathscr{I}^{\prime}\right) \rightarrow\left(A_{i}, \mathscr{I}_{i}\right)$. Since $A$ is the direct product of the $A_{i}$ and each $f_{i}$ is an MV-homomorphism of $A^{\prime}$ to $A_{i}$ we know there is a unqiue MV-homomorphism $g: A^{\prime} \rightarrow A$ such that for each $i \in I$, the diagram

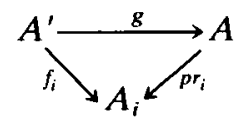

commutes, i.e. $\mathrm{pr}_{i} g=f_{i}$.

Now let $P \in \mathscr{I}$. Then for some $i \in I$ and $Q \in \Phi_{i}$, we have $P=\operatorname{Ov}(Q)$. Thus $g^{-1}(P)=g^{-1}(\operatorname{Ov}(Q))=g^{-1} \operatorname{pr}_{i}^{-1}(Q)=\left(\mathrm{pr}_{i} g\right)^{-1}(Q)=f_{i}^{-1}(Q) \in \mathscr{I}^{\prime}$ since $f_{i}$ is an $\mathscr{E}_{\mathrm{MV}}$ morphism. Hence $g:\left(A^{\prime}, \mathscr{I}^{\prime}\right) \rightarrow(A, \mathscr{I})$ is an $\mathscr{E}_{\mathrm{MV}}$-morphism and we see that $(A, \mathscr{I})$ is the product, $\prod_{i \in I}\left(A_{i}, \mathscr{I}_{i}\right)$. We shall show that $\gamma$ commutes with $\Pi$, i.e.:

THEOREM 2.1.

$$
\gamma\left(\prod_{i \in I}\left(A_{i}, \mathscr{I}_{i}\right)\right) \cong \prod_{i \in I} \gamma\left(A_{i}, \mathscr{I}_{i}\right)
$$

In the above notation this is $[A]_{\mathscr{g}} \cong \prod_{i \in I}\left[A_{i}\right]_{\mathscr{S}_{i}}$. First we require.

Lemma 2.1. Let $(A, \mathscr{I}),\left(A_{i}, \mathscr{I}_{i}\right) \in \mathscr{E}_{\mathrm{MV}}$ with $\mathscr{I}=\left\{P \mid\right.$ for some $\left.Q \in \bigcup_{i \in I} \mathscr{I}_{i}, P=\operatorname{Ov}(Q)\right\}$. Then, if $a, b \in A$, we have $[a]_{\mathscr{G}}=[b]_{\mathscr{S}}$ iff, for each $i \in I,\left[a_{i}\right]_{\mathscr{F}_{i}}=\left[b_{i}\right]_{\mathscr{F}_{i}}$.

Proof. Suppose $[a]_{\mathscr{S}}=[b]_{\mathscr{P}}$. Let $i \in I$ and let $Q \in \mathscr{I}_{i}$. Assume $a_{i} \in Q$. Let $P=\operatorname{Ov}(Q)$. Then $P \in \mathscr{I}$ and $a \in P$. Thus $b \in P$, so $b_{i} \in Q$. By symmetry we have $\left[a_{i}\right]_{\Phi_{i}}=\left[b_{i}\right]_{\mathscr{S}_{i}}$. Conversely suppose that $\left[a_{i}\right]_{\mathscr{I}_{i}}=\left[b_{i}\right]_{\mathscr{F}_{i}}$ for each $i \in I$. ket $P \in \mathscr{I}$ and suppose $a \in P$. For some $i_{0} \in I$ and some $Q \in \mathscr{I}_{i_{0}}$ we have $P=\operatorname{Ov}(Q)$. Thus $a_{i} \in Q$; hence $b_{i_{0}} \in q$ and so $b \in P$. By symmetry we conclude $[a]_{\mathscr{S}}=[b]_{\mathscr{F}}$.

Proof of Theorem 2.1. Let $\left(A, \mathscr{I}^{\prime}\right)=\prod_{i \in I}\left(A_{i}, \mathscr{I}_{i}\right)$. Define $h:[A]_{\mathscr{I}} \rightarrow \prod_{i \in I}\left[A_{i}\right]_{\Phi_{i}}$ by $h\left([a]_{\mathscr{\Phi}}\right)=\left\langle\left[a_{i}\right]_{\Phi_{i}}\right\rangle$ where $\left\langle\left[a_{i}\right]_{\mathscr{\Phi}_{i}}\right\rangle$ is that element of $\prod_{i \in I}\left[A_{i}\right]_{\mathscr{\Phi}_{i}}$ whose $i$ th component is $\left[a_{i}\right]_{\mathscr{I}_{i}}$. By Lemma $2.1 h$ is well defined and bijective. It is straight forward to verify that $h$ preserves the lattice operations; hence $h$ is an isomorphism.

In the sequel, given $\left(A_{i}, \mathscr{I}_{i}\right), i \in I$, with each $\mathscr{I}_{i}=\operatorname{spec} A_{i}, \mathscr{I}$ will be called the over-family of prime ideals of $A$ and will be denoted by $\operatorname{Ov}(\mathrm{A})$. 
Corollary 2.1. Given MV-algebras $A_{i}, i \in I, A=\prod_{i \in I} A_{i}$, we have $[A]_{\mathrm{Ov}(A)} \cong \prod_{i \in I}\left[A_{i}\right]$.

We want now to examine the special case when $I$ is finite. First, two preliminaries.

Proposition 2.1. Let $P$ be a prime ideal in $A=\prod_{i \in l} A_{i}$. Then $\operatorname{pr}_{k}(P) \neq A_{k}$ for at most one $k \in I$.

Proof. Let $i, k \in I, i \neq k$ and suppose $\operatorname{pr}_{k}(P) \neq A_{k}, \operatorname{pr}_{i}(P) \neq A_{i}$. Let $\delta_{i} \in A$ be such that the $i$ th component of $\delta_{i}$ is 1 and the $j$ th component, $j \neq i$, is 0 . Similarly for $\delta_{k}$. Clearly $\delta_{i} \wedge \delta_{k}=0$; thus $\delta_{i} \wedge \delta_{k} \in P$ so either $\delta_{i} \in P$ or $\delta_{k} \in P$. But then $1 \in \operatorname{pr}_{i}(P)$ or $1 \in \operatorname{pr}_{k}(P)$ which is impossible.

Proposition 2.2. If $I$ is finite and $P \subseteq A=\prod_{i \in I} A_{i}$ is a prime ideal of $A$, then there is exactly one $h \in I$ with $\operatorname{pr}_{h}(P) \neq A_{h}$.

Proof. We known there is at most one such $h$. Suppose that $\operatorname{pr}_{i}(P)=A_{i}$ for each $i \in I$. Choose $q_{i} \in P$ such that $\operatorname{pr}_{i}\left(q_{i}\right)=1$. Then $q=\sum_{i \in I} q_{i} \in P$, and $q=1$, absurd.

Theorem 2.2. If $I$ is finite, $A=\prod_{i \in I} A_{i}$, then $\operatorname{Ov}(A)=\operatorname{Spec} A$.

Proof. Let $P \in \operatorname{Spec} A$. By Proposition 2.2 there is a unique $h \in I$ with $\operatorname{pr}_{h}(P) \neq A_{h}$. Let $Q=\operatorname{pr}_{h}(P)$ and $Q^{\prime}=\operatorname{pr}_{h}^{-1}(Q)$. Then $Q^{\prime}$ is a proper ideal of $A, P \subseteq Q^{\prime}$. Since $Q \in \operatorname{Spec} A_{h}, Q^{\prime} \in \operatorname{Ov}(A)$. Let $a \in Q^{\prime}$. For $i \neq h, \operatorname{pr}_{i}(P)=A_{i}$ so we can find a $u_{i} \in P$ with $\operatorname{pr}_{i}\left(u_{i}\right)=\operatorname{pr}_{i}(a)$. For $h$ we know that $\operatorname{pr}_{h}(a) \in Q$ so we can find a $u_{h} \in P$ with $\operatorname{pr}_{h}\left(u_{h}\right)=$ $\operatorname{pr}_{h}(a)$. Let $u=\sum_{i \in I} \delta_{i} u_{i}$. Then $u \in P$. Now if $j \in I$ we have $\operatorname{pr}_{j}(u)=\sum_{i \in I} \operatorname{pr}_{j}\left(\delta_{i}\right) \operatorname{pr}_{j}\left(u_{i}\right)=$ $\operatorname{pr}_{j}\left(u_{i}\right)=\operatorname{pr}_{j}(a)$. Thus $u=a$ so $a \in P$; that is $Q^{\prime}=\operatorname{Ov}(Q)=P$ so $P \in \operatorname{Ov}(A)$.

Corollary 2.2. If $I$ is finite and $\mathscr{I}_{i}=\operatorname{Spec} A_{i}$ for each $i \in I$, then $[A] \cong \prod_{i \in I}\left[A_{i}\right]$.

Proof. By Theorem 2.2 and Corollary 2.1 .

Corollary 2.3. If $A$ is a finite MV-algebra, then $\operatorname{Spec} A$ has a base of clopen sets.

Proof. By [5, Corollary 2.7], $A=\prod_{i \in I} A_{i}$, where $A_{i}$ is a linearly ordered MV-algebra for every $i \in I$. Then, by Corollary $2.2,[A] \simeq \prod_{i \in I}\left[A_{i}\right]=\prod_{i \in I}\{0,1\}$, i.e. $[A]$ is a boolean algebra. Thus $\operatorname{Spec} A$, which is homeomorphic to $\operatorname{Spec}[A]$, has a base of clopen sets.

3. We would like now to examine the behavior of $\gamma$ with respect to subobjects, i.e. monomorphisms. It is easy to see in general that $\gamma$ does not preserve monomorphisms. For example if $\mathscr{I}$ is any proper subset of $\operatorname{Spec} A$ we have an $\mathscr{E}_{\mathrm{MV}}$ monomorphism $i:(A, \operatorname{Spec} A) \rightarrow(A, \mathscr{I})$ where $i$ is the identity map. But, in general, $[x] \rightarrow[x]_{\mathscr{S}}$ will not be one-one. For some subobjects however, $\gamma$ will preserve monicity. In particular we will show if $A$ is a subalgebra of $B$ then $[A]$ is isomorphic to a sublattice of $[B]$.

To begin, let $\left(A_{1}, \mathscr{I}_{1}\right),\left(A_{2}, \mathscr{I}_{2}\right)$ be in $\mathscr{E}_{\mathrm{MV}}$. We call $\left(A_{1}, \mathscr{I}_{1}\right)$ a full subobject of $\left(A_{2}, \mathscr{I}_{2}\right)$ if there is a monomorphism $f:\left(A_{1}, \mathscr{I}_{1}\right) \rightarrow\left(A_{2}, \mathscr{I}_{2}\right)$ such that $f^{-1}\left(\mathscr{I}_{2}\right)=\mathscr{I}_{1}$, i.e. if $P \in \mathscr{I}_{1}$ there is a $Q \in I_{2}$ with $f^{-1}(Q)=P$. 
THEOREM 3.1. If $\left(A_{1}, \mathscr{I}_{1}\right)$ is a full subobject of $\left(A_{2}, \mathscr{I}_{2}\right)$ then there is an injective homomorphism of $\left[A_{1}\right]_{\mathcal{F}_{1}}$ into $\left[A_{2}\right]_{\mathscr{F}_{2}}$.

Proof. There is a monomorphism $f:\left(A_{1}, \mathscr{I}_{1}\right) \rightarrow\left(A_{2}, \mathscr{I}_{2}\right)$. Thus $f: A_{1} \rightarrow A_{2}$ is an MV-monomorphism and $f^{-1}\left(\mathscr{I}_{2}\right)=\mathscr{I}_{1}$. We have a homomorphism $\gamma(f):\left[A_{1}\right]_{\mathscr{F}_{1}} \rightarrow\left[A_{2}\right]_{\mathscr{I}_{2}}$ where $\gamma(f)\left([x]_{\mathscr{S}_{1}}\right)=[f(x)]_{\mathscr{S}_{2}}$. Suppose $[f(x)]_{\mathscr{S}_{2}}=[f(y)]_{\mathscr{F}_{2}}$. Let $P \in \mathscr{I}_{1}$. Assume $x \in P$. Now $P=f^{-1}(Q)$ for some $Q \in \Phi_{2}$. So $f(x) \in Q$. Thus $f(y) \in Q$ and so $y \in f^{-1}(Q)=P$. By symmetry we have $[x]_{\mathscr{P}_{1}}=[f(x)]_{\mathscr{S}_{2}}$ and so $\gamma(f)$ is one-one.

Now let $A \subseteq B, A$ a subalgebra of $B$. We have the inclusion map $i: A \rightarrow B, i(x)=x$. If $Q \in \operatorname{Spec} B$ then $i^{-1}(Q)=A \cap Q \in \operatorname{Spec} A$. Thus $i^{-1}(\operatorname{Spec} B) \subseteq \operatorname{Spec} A$ so $i:(A, \operatorname{Spec} A) \rightarrow(B, \operatorname{Spec} B)$ is a subobject of $(B, \operatorname{Spec} B)$.

THeOREM 3.2. If $A, B$ are MV-algebras, $A$ a subalgebra of $B$, then

$$
\operatorname{Spec} A=\{A \cap Q \mid Q \in \operatorname{Spec} B\} .
$$

Proof. Clearly $\{A \cap Q \mid Q \in \operatorname{Spec} B\} \subseteq \operatorname{Spec} A$. Let $P \in \operatorname{Spec} A$. Let $H$ be the ideal in $B$ generated by $P$. Let $G$ be the lattice-filter in $B$ generated by $A-P$. If $x \in H \cap G$ then there is a $p \in P$ with $x \leq p$ and a $z \in A-P$ with $z \leq x$. This implies $z \leq p$, so $z \in P$ which is impossible. So $H \cap G=\varnothing$. By [7, Theorem 2.5], there is a prime ideal $Q \in \operatorname{Spec} B$ with $H \subseteq Q, Q \cap G=\varnothing$. $A=P \cup(A-P)$, so $A \cap Q=P \cap Q=P$ since $P \subseteq Q$ and $Q \cap(A-P)=\varnothing$.

Corollary 3.1. If $A$ is a subalgebra of $B$ then $(A, \operatorname{Spec} A)$ is a full subobject of $(B, \operatorname{Spec} B)$.

Proof. Let $i: A \rightarrow B$ be the inclusion map. If $P \in \operatorname{Spec} A$ then by the above theorem, $P=i^{-1}(Q)$ for some $Q \in \operatorname{Spec} B$.

CoROLlaRY 3.2. If $A$ is a subalgebra of $B$ there is an injective homomorphism of $[A]$ into $[B]$.

Proof. Clear from the above corollary and Theorem 3.1.

4. Given that not every lattice in $\mathscr{D}$ is in the range of $\gamma$ it becomes pertinent to know which lattices are. We know that some countable chains lie in the range of $\gamma$. In this section we show the same is true for the chain $[0,1]$, in fact for any complete bonded chain.

To this end let $\mathbb{N}$ be the set of positive integers, $\mathscr{F}$ a maximal filter in $2^{\mathbb{N}}$ that contains all cofinite subsets of $\mathbb{N}$. Let $A$ be the ultrapower $[0,1]^{\mathbb{N}} / \mathscr{F}$. Then $A$ is a linearly ordered MV-algebra. For each $r \in[0,1]$ let $\tau_{r}$ be the element of $A$ determined by the sequence $\left\langle r, r^{2}, r^{3}, \ldots\right\rangle \in[0,1]^{\mathbb{N}}$. We then have

Proposition 4.1. Let $r, s \in[0,1), 0<r<s \leq 1$. Let $P_{r}, P_{s}$ be the ideals of $A$ generated by $\tau_{r}, \tau_{s}$ respectively. Then $P_{r} \subseteq P_{s}$ and $\tau_{s} \notin P_{r}$.

Proof. Since $0<r<s$ we have $1<s / r$. Let $h$ be any positive integer. Then there is a least integer $n_{0} \in \mathbb{N}$ such that $h<(s / r)^{n}$ for all $n \geq n_{0}$. Hence $\left\{n \mid h r^{n}<s^{n}\right\}=\{n \mid n \geq$ $\left.n_{0}\right\} \in \mathscr{F}$. Thus $h \tau_{r}<\tau_{s}$ and so $\tau_{s} \notin P_{r}$. Clearly $\tau_{r}<\tau_{s}$ so $\tau_{r} \in P_{s}$; thus $P_{r} \subseteq P_{s}$. 
Let $s \in[0,1)$. Set $P_{s}^{\prime}=\bigcap_{s<r} P_{r}$. From the above we have $P_{s} \subseteq P_{s}^{\prime}$. Since $A$ is linearly ordered all of its ideals are prime, if proper. Thus each $P_{s}^{\prime}, s \in[0,1)$, is a prime ideal. Let $\mathscr{I}=\left\{P_{s}^{\prime} \mid s \in[0,1)\right\}$. Then $\mathscr{I} \neq \varnothing$, and $\mathscr{I} \subseteq \operatorname{Spec} A$.

Proposition 4.2. For each $x \in A$ there is an $s \in[0,1]$ with $[x]_{\mathscr{F}}=\left[\tau_{s}\right]_{\mathscr{F}}$.

Proof. If $x \notin P_{r}$ for any $r \in[0,1)$ then $x \notin P_{s}^{\prime}$ for any $s \in[0,1)$. Thus $[x]_{\mathscr{S}}=1=\left[\tau_{1}\right]$.

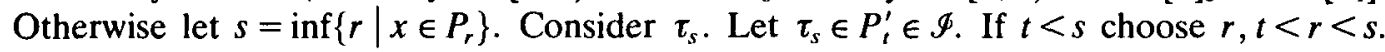
By Proposition $4.1 \tau_{s} \notin P_{r}$, hence $\tau_{s} \notin P_{t}^{\prime}$. Thus $s \leq t$. If $s=t$ then for $t<r \leq 1$ we have $x \in P_{r}$ and so $x \in \bigcap_{t<r} P_{r}=P_{t}^{\prime}$. If $s<t$ choose $r, s<r<t$ with $x \in P_{r}$. Since $P_{r} \subseteq P_{t} \subset P_{t}^{\prime}$ we

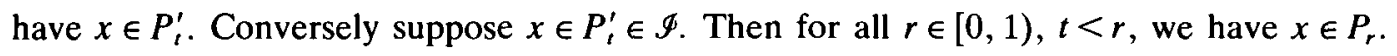
Hence for all $r \in[0,1), t<r$, we have $s \leq r$, so $\tau_{s} \in P_{r}$. Thus $\tau_{s} \in \bigcap_{t<r} P_{r}=P_{t}^{\prime}$. Hence $[x]_{\mathscr{S}}=\left[\boldsymbol{\tau}_{s}\right]_{\mathscr{S}}$.

From the above proposition we see that $[A]_{\mathscr{A}}=\left\{\left[\tau_{s}\right]_{\mathscr{S}} \mid s \in[0,1]\right\}$. Since it is evident that $\left[\tau_{s}\right] \leftrightarrow s$ is an order preserving bijection we obtain

THEOREM 4.3. There is a linearly ordered MV-algebra $A$ and non-empty subset $\mathscr{I} \subseteq \operatorname{Spec} A$ such that $[A]_{\mathscr{F}} \cong[0,1]$.

To extend the above result to any complete bounded chain we set some premises.

Let $\mathscr{L}$ be a first order language for the theory of MV-algebras. Extend $\mathscr{L}$ to $\mathscr{L}^{+}$by adding constant symbols, $c_{r}$, one for each $r \in \mathscr{C}$ where $\mathscr{C}$ is a given complete bounded chain. Let $\Delta_{1}$ be the first order axioms for linearly ordered MV-algebras. Let $\Delta_{2}=\left\{n c_{r}<c_{s} \mid n=1,2 \ldots ; r, s \in \mathscr{C}, r<s\right\}$. Let $\Delta=\Delta_{1} \cup \Delta_{2}$. We now have

Proposition 4.3. Every finite subset $\Delta^{\prime}$ of $\Delta$ has a model.

Proof. Let $c_{r_{1}}, c_{r_{2}}, \ldots, c_{r_{n}}$ be the constant symbols occurring in the formulas of $\Delta^{\prime}$ (we can suppose $r_{n}<r_{n-1} \ldots<r_{1}$ ).

Let $E_{n}$ be a subalgebra of a proper ultrapower $[0,1]^{*}$ of $[0,1]$ generated by $\varepsilon, \varepsilon^{2}, \ldots \varepsilon^{n}$ where $\varepsilon$ is a non-zero infinitesimal. Then interpreting $c_{r_{k}}$ by $\varepsilon^{k} E_{n}$ becomes a model for $\Delta^{\prime}$.

Thus, by the compactness theorem, we have the following

Corollary 3.3. $\Delta$ has a model.

THEоRем 4.4. Let $\mathscr{C}$ be a complete bounded chain. Then there exist an MV-algebra $\mathscr{A}$ and a family $\mathscr{I}$ of prime ideals of $\mathscr{A}$ such that $[\mathscr{A}]_{\mathscr{F}}$ is isomorphic to $\mathscr{C}$.

Proof. Let $\mathscr{A}=\left\langle A,+, .,-, 0,1\left\{a_{r}: r \in \mathscr{C}\right\}\right\rangle$ be a model of $\Delta$, by Corollary 3.3 . Moreover if $r<s$, then, for any positive $n, n a_{r}<a_{s}$. Hence if we set $P_{r}=\left\langle a_{r}\right\rangle$, the principal ideal generated by $a_{r}$, we get prime ideals $P_{r} \subset P_{s}$ if and only if $r<s, r, s \in \mathscr{C}$. For each $x \in A$ let us define the set $B(x)=\left\{r \in \mathscr{C} \mid x \in P_{r}\right\}$, and set $m(x)=\inf B(x)$. Consider the map $f$ defined by

$$
f:[x] \in[A] \rightarrow f([x])=m(x) .
$$


Then:

(i) $f$ is well defined: indeed if $x \equiv y \quad(\operatorname{Spec} A)$ then $B(x)=B(y)$, which implies $m(x)=m(y)$.

(ii) $f$ is a homomorphism: indeed it is increasing because if $[x]<[y]$ then $B(y) \subseteq B(x)$ so $m(x) \leq m(y)$.

(iii) $f$ is onto: let $r \in \mathscr{C}$, then we have that $r=\min B\left(a_{r}\right)=m\left(a_{r}\right)$. Thus, by [2, Theorem 3.1], there is a set $\mathscr{I} \subseteq \operatorname{Spec} A$ such that $[A]_{\mathscr{I}} \cong \mathscr{C}$.

\section{REFERENCES}

1. L. P. Belluce, Semisimple algebras of infinite valued logic and bold fuzzy set theory, Canad. J. Math., 38 (1986), 1356-1379.

2. L. P. Belluce, A. Di Nola and A. Lettieri, On some lattices quotients of MV-Algebras, Ricerche di Matemat. 39 (1990), 41-59. 467-490.

3. C. C. Chang, Algebraic analysis of many valued logics, Trans. Amer. Math. Soc. 88 (1958),

4. C. C. Chang, A new proof of the completeness of the Lukasiewicz axioms, Trans. Amer. Math. Soc. 93 (1959), 74-80.

5. R. Cignoli, Complete and atomic algebras of the infinite-valued Lukasiewicz logic, unpublished paper.

6. R. Cignoli, A. Di Nola and A. Lettieri, Priestley duality and quotient lattices of many-valued algebras, Rend. Circ. Matem. Palermo, to appear.

7. C. S. Hoo, Mv-algebras, ideals and semisimplicity, Math. Japon 34 (1989), 563-583.

8. U. Maddana Swany and D. Viswanadha Rajn, A note on maximal ideal spaces of distributive lattices, Bull. Calcutta Math. Soc., 80 (1988) 84-90.

9. N. G. Martinez, Priestley duality for Wajesberg algebras, Studia Logica, 49 (1990), 31-46.

10. D. Mundici, Interpretation of AF $C^{*}$-algebras in Lukasiewicz sentential calculus, $J$. Functional Analysis 65 (1986) 15-63.

11. A. J. Rodriguez, Un estudio algebraico de los calculos proposicionales de Lukasiewicz, thesis, Universidad de Barcelona, 1980. $567-583$.

12. A. Romanoskwa and T. Traczyk, On commutative BCK-algebras, Math. Japon 25 (1980),

L. P. Belluce

Department of Mathematics

University of British Columbia

VANCOUVER, B.C.

CANADA

\author{
A. Di Nola and A. Lettieri \\ Istituto di Matematica \\ Facolta' di Architettura \\ UNIVERSITA' DI NAPOLI \\ 80134 Via MonTEOLIVETo N. 3 \\ Napoli, Italy
}

